\title{
Feasibility and outcome of laparoscopic management of complicated diverticulosis
}

\author{
Nabil Shedid, FRCS; Gamal I. El-Habbaa, MD; Hazem Sobieh, MD \\ Departments of General Surgery, Benha University, Egypt.
}

\begin{abstract}
Objectives: To evaluate the surgical feasibility and short-term outcome of laparoscopic management of complicated cases of diverticular disease.

Patients and methods: The study included 12 patients; 9 males and 3 females with mean age of $57 \pm 5.8$ years. All patients had preoperative colonoscopy and CT imaging to assure diagnosis. All patients had exploratory laparoscopy to either proceed or convert to open laparotomy and in all cases, a sigmoidectomy was performed with primary intracorporeal anastomosis using a circular end-to-end stapling device. Intraoperative data including duration of surgery, estimated blood loss, length of excised specimen, type and number of managed diverticular complications and number of cases converted to open surgery and time till first ambulation, time till first oral intake, length of hospital stay and postoperative morbidity and mortality were recorded.

Results: Eight cases had successful laparoscopic management and 4 patients were converted to open surgery with a conversion rate of 33.3\%. Exploratory laparoscopy detected variant pathologies in the same case; 7 patients had diverticular abscess that was drained with peritoneal lavage and primary colectomy with anastomosis was performed. Two cases had single colo-vesical fistula that was closed with inverting burse-string suture, then colectomy was conducted successfully. Two females had colosalpingeal fistula; left salpingectomy was performed. Mean operative time was $180.6 \pm 32.9$ minutes and mean operative blood loss was $89 \pm 11.6 \mathrm{cc}$, mean time till $1^{\text {st }}$ mobilization and till having audible intestinal sounds was $13.2 \pm 3.1$ and $2.6 \pm 0.7$ days, respectively. Mean time till 1st oral intake was $3.2 \pm 0.8$ days and length of hospital stay was $6.2 \pm 1.2$ days. One patient required re-admission for signs of acute abdomen, clinical and CT examination revealed a localized left iliac fossa collection indicating anastomotic line leakage and the patient had open peritoneal lavage and diversion colostomy.

Conclusion: Laparoscopic management of complicated diverticulitis is feasible and safe approach provided proper surgical decision was adequately taken concerning to proceed or not after laparoscopic exploration.
\end{abstract}

Key words: Complicated diverticulitis, laparoscopic approach, colo-vesical fistula, colosalpingeal fistula.

\section{Introduction:}

Diverticulosis is considered to be mainly a problem of old age, with a prevalence of $35-50 \%$. About $10-25 \%$ of patients with diverticulosis will develop diverticular disease compilation in their lifetime. The clinical presentation of diverticular disease depends on the severity of the inflammatory process and whether complications are present. Complicated diverticulitis refers to the presence of perforation, obstruction, bleeding and abscess or fistula formation. Between 25 and $55 \%$ of the patients with complicated diverticulitis will require surgery during their initial hospitalization. 1,2

The first attack of uncomplicated diverticulitis is treated conservatively. Sigmoid resection is indicated for 
recurrent diverticulitis, in patients with manifest stenosis or fistula and for such emergencies as perforation, ileus or bleeding. Early surgery after the first episode is recommended for patients under 50 years of age, or immunocompromised patients. This is particularly true for patients with radiological signs of severe diverticulitis. ${ }^{3}$

Elective laparoscopic resection for recurrent, uncomplicated diverticulitis of the sigmoid is considered safe and effective and may fare better than its conventional counterpart not only in short-term outcome including preservation of the abdominal wall and shorter disability, but also in the long term as decreased rates of late symptomatic small bowel obstruction. Five-year recurrence rates show that a laparoscopic or conventional access is unlikely to have an impact, provided that the oral bowel end is anastomosed to the proximal rectum rather than to the distal sigmoid. 4,5

Little data exist on complicated cases and multiple non-randomized studies indicated that the role of laparoscopic surgery should be limited to resection for uncomplicated diverticulitis of the sigmoid performed by adequately trained surgeons. Benefits can be expected with this procedure, provided that indications for surgery are not influenced by the mode of access and that postoperative complication rates remain within the range of that for traditional colorectal surgery. 6,7

Elective sigmoid resection became a laparoscopic procedure that if properly carried out, the operation affects a definitive cure, the morbidity and mortality of the operation is low, and re-operations for recurrent diverticulitis are the exception. In the emergency situation a two-stage procedure is often necessary. The superiority of laparoscopy should be proven by measuring health-related and patientcentered outcome rather than surrogate endpoints. Areas of concern include replacing a conventional resection with laparoscopic suture, drainage, and colostomy in patients with free perforation and peritonitis. 8,9

The current study aimed to evaluate surgical feasibility and short-term outcome of laparoscopic management of complicated cases of diverticular disease.

\section{Patients and methods:}

The present study was conducted at General Surgery Department, Benha University Hospital since June 2007 till October 2010. The study intended to include complicated diverticulitis patients, patients with urological complaint were subjected to urological diagnosis workup, those who had anemia were investigated for its cause with regard to malignancy especially if anemia was associated with weight loss. Patients presenting with manifestations of acute diverticulitis, malignancy, other indications for left colectomy, or who had scar of previous abdominal open surgery hampering peritoneal insufflations were excluded of the study

All surgeries were conducted under general anesthesia. Patients were positioned in a modified lithotomy position, with table adjusted to $15^{\circ}$ Trendelenburg position and slightly to the right side, but during left flexure mobilization, the table was kept in an anti-Trendelenburg position to move the small bowel toward the pelvis. Four trocars were used, the first trocar was introduced 4-cm above the umbilicus by an open Hasson technique, and a pneumoperitoneum was insufflated to $12-14 \mathrm{mmHg}$. The $2^{\text {nd }}$ and $3^{\text {rd }}$ trocars were placed under direct vision at the left hypochondrial and right lumbar regions, respectively. The 4 th trocar was placed $4-\mathrm{cm}$ above the pubic bone. The laparoscope was introduced through the supraumbilical trocar and exploratory laparoscopy was conducted.

For laparoscopic colonic resection, sigmoid colon mobilization began by dividing natural attachments to the lateral abdominal wall, retroperitoneum, and other adjacent organs using Harmonic scissor and continues along the peritoneal reflection. Then, the phrenocolic and splenocolic attachments were divided to mobilize the splenic flexure of the colon in some cases to create a tension-free anastomosis. The dissection was completed with mobilization of the descending colon, the sigmoid tract, and the upper third of the rectum, which 
was divided by a linear stapler cutter. The suprapubic trocar opening was extended for about a 3-6 $\mathrm{cm}$ and minilaparotomy was performed for extraction of the sigmoid colon for signoidectomy. A 29-head circular stapler was inserted in the colonic stump and tied with a 2.0 polypropylene purse-string suture. The colonic stump was returned to the peritoneal cavity, the minilaparotomy incision was closed in layers and pneumoperitoneum was recreated. A circular end-to-end stapler was advanced via the anus with its pin pushed directly above the center of the stapler line at the upper third of the rectum, and a double-stapled primary intracorporeal anastomosis was obtained, Figure(1a\&b). Paraanastomotic areas were drained, the trocars were removed, and the deep fascias of the ports were closed

\section{Patients' evaluation and data collection}

1-Preoperative evaluation included personal data; age and sex, constitutional data including body weight, height and calculation of body mass index (BMI). Patients' clinical data including American Society of Anesthesiologists (ASA) grade, preoperative antibiotic use, and preoperative colonoscopic and CT findings were determined.

2-Intraoperative data included duration of surgery, estimated blood loss, length of excised specimen, type and number of managed diverticular complications and number of cases converted to open surgery.

3-Postoperative data included time till first ambulation, time till first oral intake, length of hospital stay and postoperative morbidity and mortality.

\section{Results:}

The study included 12 patients; 9 males and 3 females with mean age of $57 \pm 5.8$; range: $48-66$ years. Mean BMI was $31.7 \pm 1$; range: $30.8-34.2 \mathrm{~kg} / \mathrm{m}^{2}$. All patients had repeated courses of antibiotics; 7 patients were ASA-I grade, 3 patients were ASA-II grade and only 2 patients were ASA-III grade, Table(1).

Table (1): Patients enrollment data.

\begin{tabular}{|l|l|l|}
\hline Data & & Findings \\
\hline \multirow{2}{*}{ Age (years) } & & $57 \pm 5.8(48-66)$ \\
\hline \multirow{3}{*}{ Sex } & Males & $9(66.7 \%)$ \\
\cline { 2 - 3 } & Females & $3(33.3 \%)$ \\
\hline \multirow{3}{*}{ Body constitutional data } & Body weight $(\mathrm{kg})$ & $90.2 \pm 2.4(87-93)$ \\
\cline { 2 - 3 } & Body height $(\mathrm{cm})$ & $168.8 \pm 2.5(164-172)$ \\
\cline { 2 - 3 } & BMI $(\mathrm{kg} / \mathrm{m} 2)$ & $31.7 \pm 1(30.8-34.2)$ \\
\hline \multirow{3}{*}{ ASA grade } & ASA-I & $7(58.3 \%)$ \\
\cline { 2 - 3 } & ASA-II & $3(25 \%)$ \\
\cline { 2 - 3 } & ASA-III & $2(16.7 \%)$ \\
\hline
\end{tabular}

Data are presented as mean $\pm S D$ and numbers; ranges and percentages are in parenthesis.

BMI: body mass index.

All patients had preoperative colonoscopy that assured the diagnosis and defined the presence of single or multiple diverticular orifices, Figure(2). Colonoscopy was difficult in some cases due to colonic tortuosity and lack of distensibility. Preoperative CT imaging showed positive data indicating the diagnosis of diverticular disease with fluid
ASA: American Society of Anesthesiologists.

and air collection, Figure(3). CT examination of the two females who had laparoscopic management showed left adnexal mass in both of them and left colosalpingeal fistula with fluid collection, Figure(4).

Eight cases passed smooth intraoperative course without conversion to open surgery with a laparoscopic success rate of $66.7 \%$. 
Four patients were converted to open surgery; the first was a postmenopausal female who had multiple colo-uterine and colo-vesical fistulas with left adnexal abscess including the left fallopian tube and left ovary. Exploratory laparotomy confirmed the laparoscopic findings and assured healthy right ovary, subtotal supra-vaginal hysterectomy including the left adnexal mass with the ovary and the tube was done. Then, colectomy was conducted and the phlegmon en-bolc was removed, and no diversion was required. The patient passed smooth postoperative course and was discharged on the $17^{\text {th }}$ postoperative day without developing additional morbidities.

The second patient was a male patient in whom exploratory laparoscopy defined multiple separate points of tight adhesions between small intestine and sigmoid colon with non-adherent intervening enteric segments, this finding aroused suspicion of multiple entero-colic fistulas that was assured during laparotomy. These multiple fistulas required multiple small intestinal resections in addition to the intended colectomy and open prophylactic diverting entrostomy was performed to safeguard the performed anastomoses. This patient received postoperative care at ICU for being ASA grade III with previous history of cardiac attack and was maintained on parentral nutrition for two weeks. Then, barium follow-through was performed through the entrostomy and complete anastomotic line healing was assured and a second setting was performed for entero-anastomosis and patient was discharged after complete wound healing on the $36^{\text {th }}$ day after the first surgery.
The remaining two patients were converted to open laparotomy because of the extensive adhesions that divided the abdominal cavity into compartments, hampering advancement of the laparoscope and obscuring the field visibility, so for sake of patients' safety surgical decision was changed to open surgery. These four patients were excluded from the data collected concerning laparoscopic surgery. However, these cases indicated the efficacy of first look laparoscopy prior to surgicaldecision taking.

Exploratory laparoscopy detected varied pathologies in combination with multiplicity of pathologies in the same case; differentially, phlegmon Figure(5) was detected in six cases Figure(6) and diverticular abscess in 7 cases. Abscess' drainage Figure(7) was followed by peritoneal lavage with suction till the returning fluid was clear, then colectomy with anastomosis was performed. Two cases had single colo-vesical fistula that was dissected till exposure of fistula orifices, Figure(8). Bladder wall was closed with inverting purse-string suture placed in healthy bladder tissue and competence of orifice closure was assured by filling the bladder with methylene blue stained saline injected through the trans-urethral catheter into the bladder, then colectomy was conducted successfully. In two females who had colosalpingeal fistula; left salpingectomy was conducted but the uterus and right tube were healthy and were left undisturbed Table(2). All cases required left splenic flexure mobilization to allow free colonic handling for resection and anastomosis. Histopathological examination of resected colonic specimens showed signs of active inflammation.

Table (2): Laparoscopic operative findings.

\begin{tabular}{|l|l|}
\hline Data & Findings \\
\hline Phlegmon & $6(75 \%)$ \\
\hline Abscess & $7(87.5 \%)$ \\
\hline Fistula (Colo-vesical) & $2(25 \%)$ \\
\hline Uterine tube abscess and colosalpengeal fistula & $2(25 \%)$ \\
\hline
\end{tabular}

Data are presented as numbers; percentages are in parenthesis.

Findings were presented per the studied 8 cases irrespective of other pathologies so the total number of cases is over because multiplicity of pathologies detected. 
Mean operative time was $159.4 \pm 29.9$; range: $115-200$ minutes and mean operative blood loss was $89 \pm 11.6$; range: $70-110 \mathrm{cc}$.
The mean length of resected colon was 14 \pm 1.7 ; range: $11-16 \mathrm{~cm}$ Table(3).

\section{Table (3): Operative data.}

\begin{tabular}{|l|c|}
\hline Data & Findings \\
\hline Operative time $(\mathrm{min})$ & $159.5 \pm 29.9(115-200)$ \\
\hline Operative blood loss $(\mathrm{cc})$ & $89 \pm 11.6(70-110)$ \\
\hline Length of resected specimen $(\mathrm{cm})$ & $14 \pm 1.7(11-16)$ \\
\hline
\end{tabular}

Data are presented as mean $\pm S D$; ranges are in parenthesis.

Mean time till 1st mobilization was $13.2 \pm 3.1$; range: $10-20$ hours and mean time till having audible intestinal sounds was $2.6 \pm 0.7$; range: $2-4$ days and mean time till 1st oral intake was $3.2 \pm 0.8$; range: $2-5$ days. For both patients who had colo-vesical fistulas, urethral catheter was removed on the 6 th and $8^{\text {th }}$ day respectively, after assurance of no leakage using ascending cystogram. Mean length of hospital stay was 6.2 \pm 1.2 ; range: 5-8 days Table(4). Till home discharge, no mortality was reported and only one patient required re-admission for signs of acute abdomen. Clinical and CT examination revealed a localized left iliac fossa collection indicating anastomotic line leakage. After stabilization of general condition, the patient had laparotomy for peritoneal lavage and diversion colostomy was performed and the patient completed his postoperative care without developing additional morbidities and was asked to attend for follow-up at the outpatient clinic monthly till colostomy reversal 3 months later.

Table (4): Postoperative data.

\begin{tabular}{|l|c|}
\hline Data & Findings \\
\hline Time till 1st mobilization (hours) & $13.2 \pm 3.1(10-20)$ \\
\hline Time till have audible intestinal sounds (days) & $2.6 \pm 0.7(2-4)$ \\
\hline Time till 1st oral intake (days) & $3.2 \pm 0.8(2-5)$ \\
Length of hospital stay (day) & $6.2 \pm 1.2(5-8)$ \\
\hline
\end{tabular}

Data are presented as mean $\pm S D$; ranges are in parenthesis. 


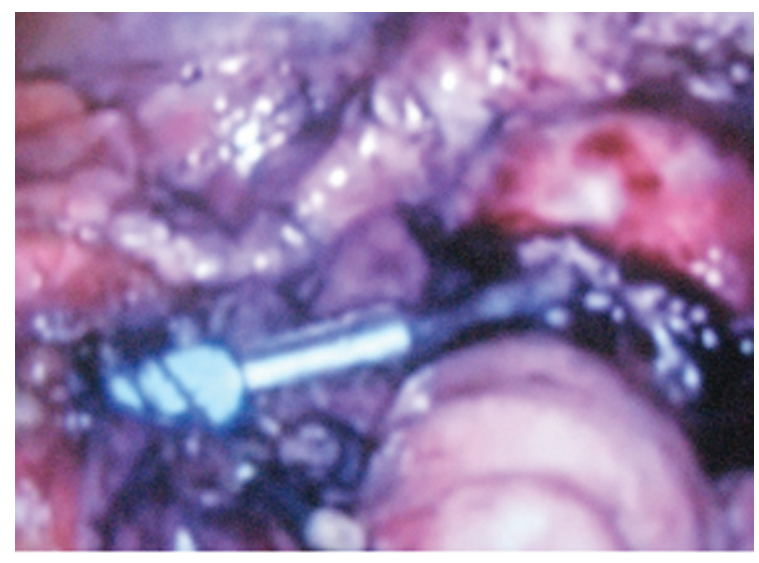

Figure (1): Shows laparoscopic colonic anastomosis.
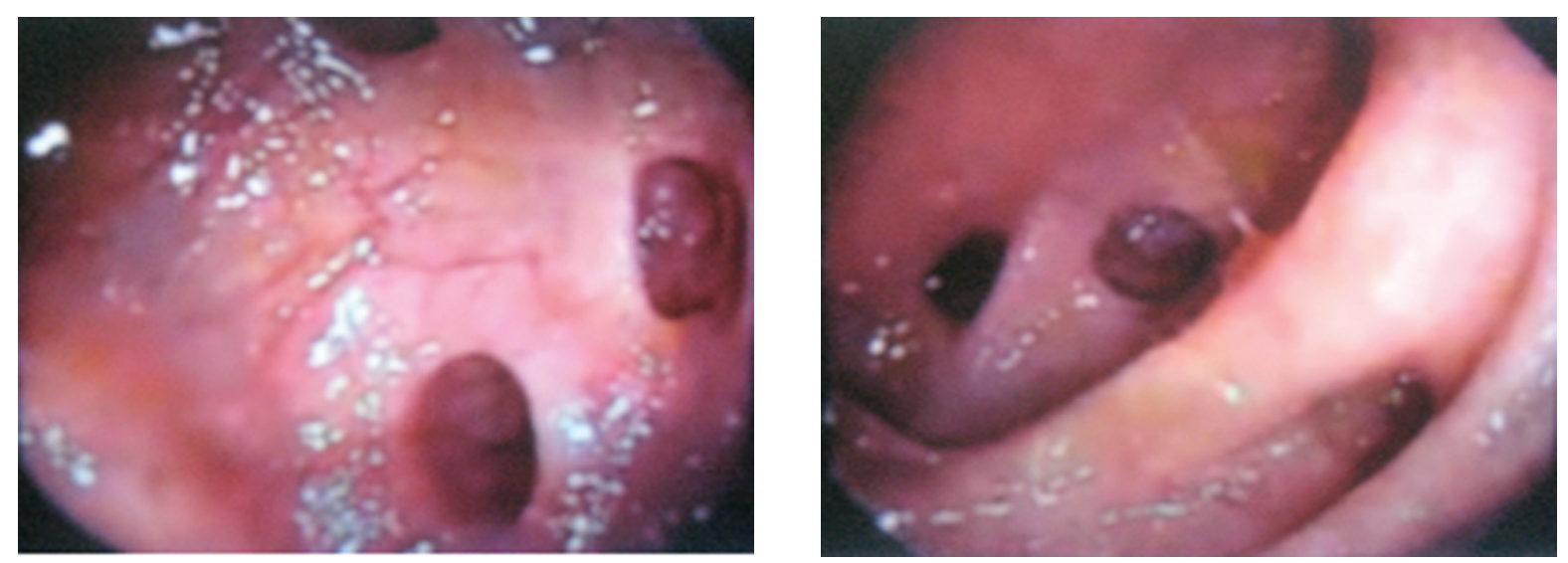

Figure (2): Shows endoscopic images of diverticuli; several diverticula are encountered, increased colonic tortuosity.
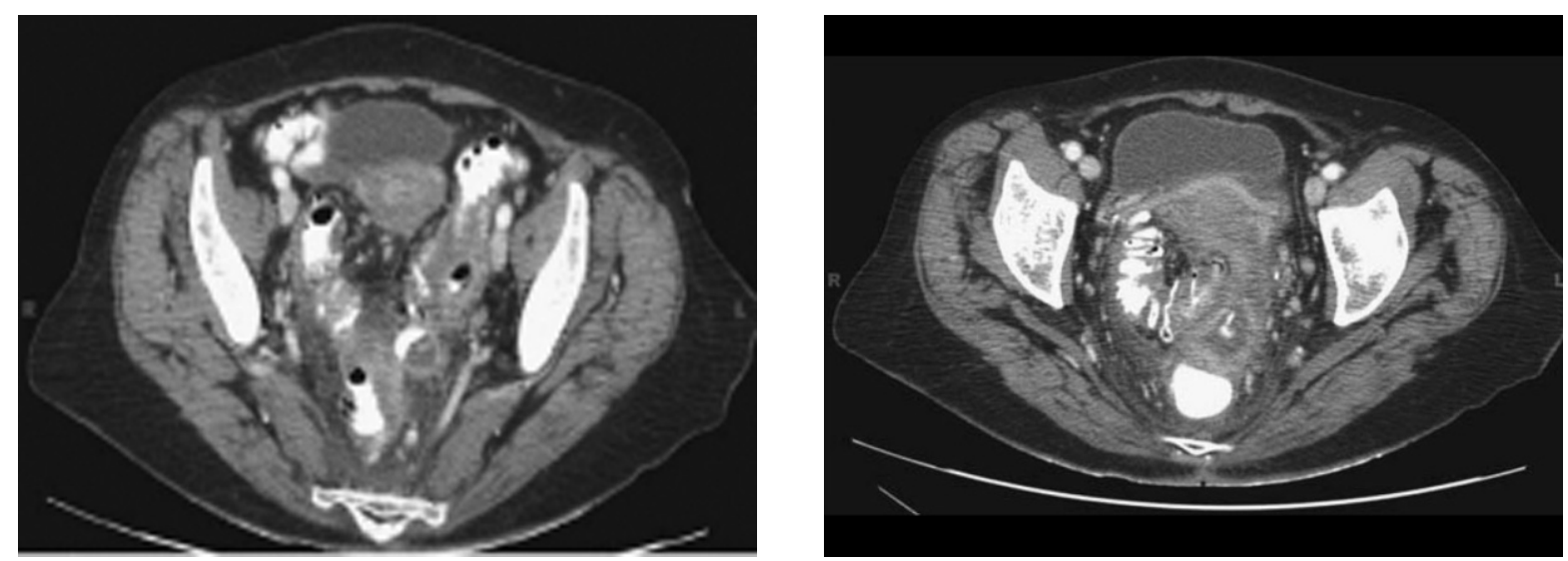

Figure (3): Shows CT scan images of a patient who presented with complicated diverticulitis and an extraluminal air and fluid collection. 

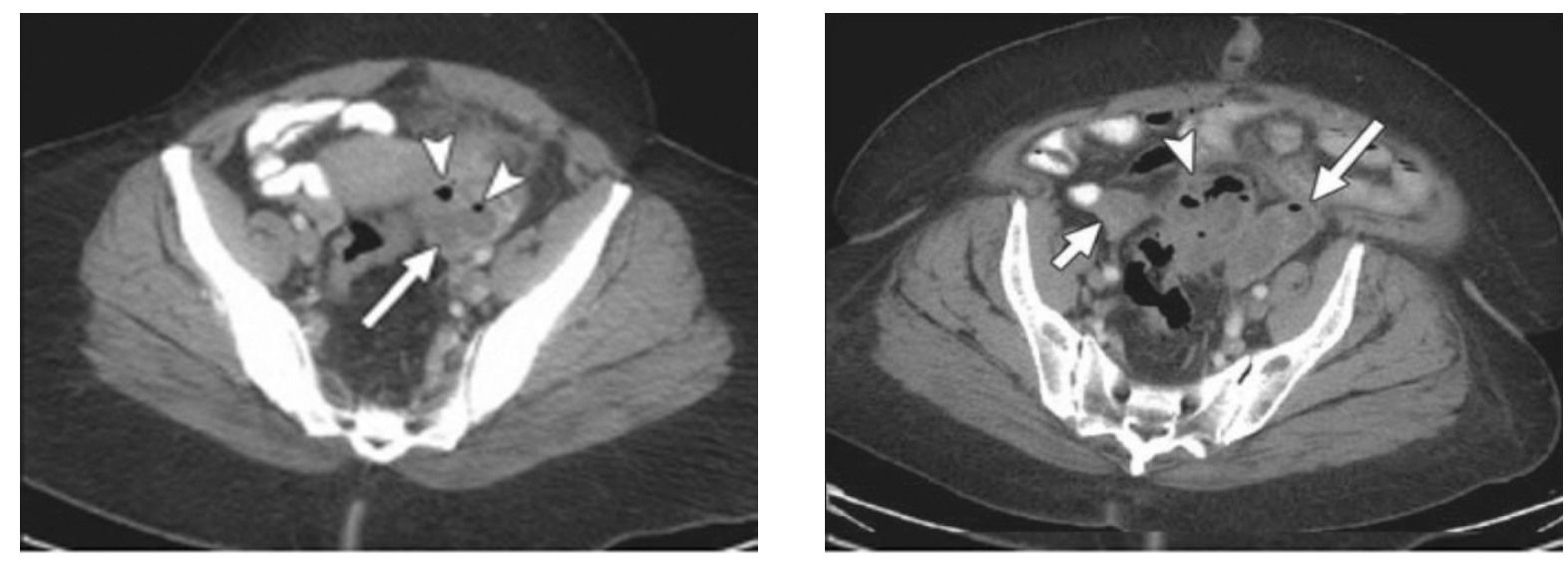

Fig. (4): Shows CT scan of a woman with sigmoid diverticulitis and colosalpingeal fistula. Axial contrast-enhanced CT scan of pelvis shows normal size of right adnexum (short arrow), enlarged left adnexum containing fluid (long arrow) and foci of gas (heads).

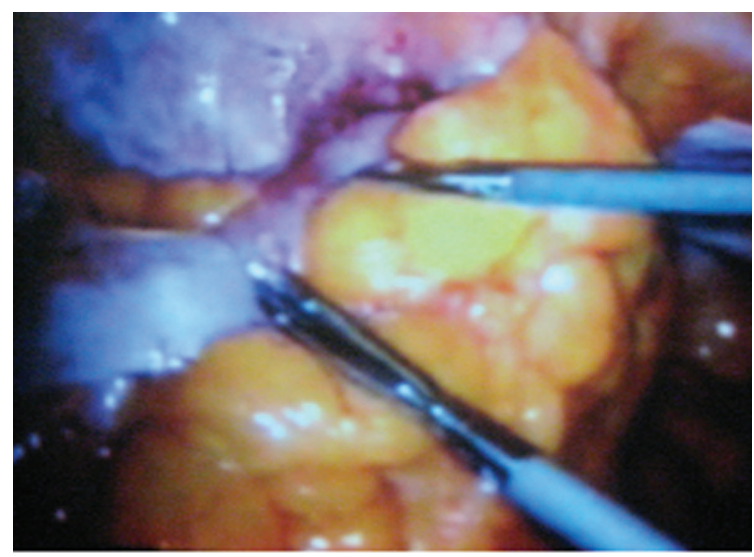

Figure (5): Showing phlegmon including the sigmoid colon and adjacent viscera.

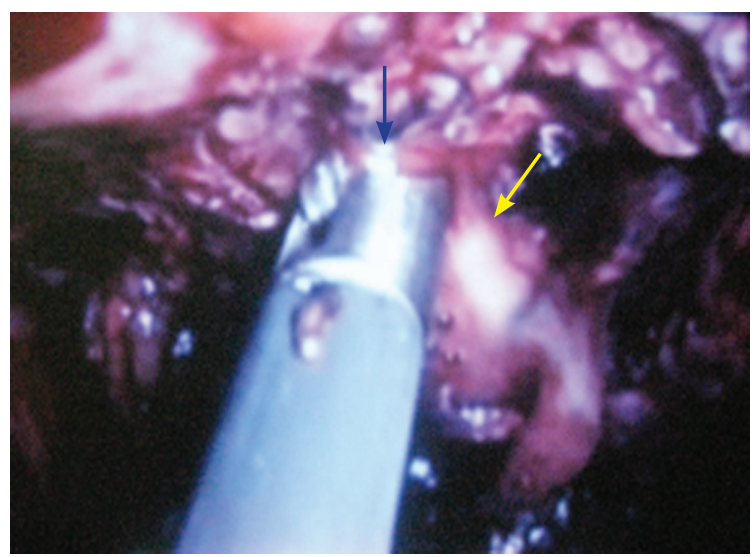

Figure (6): Showing the abscess site (blue arrow) while drained with definitely extruded pus (yellow arrow).

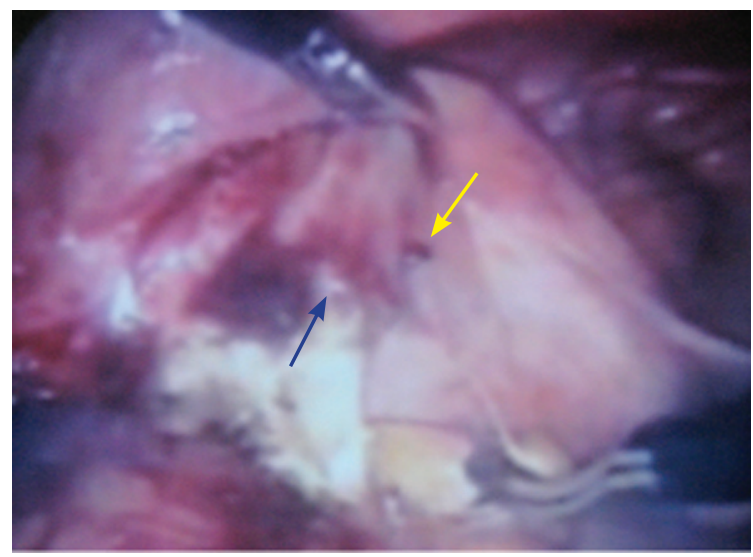

Figure (7): Showing both orifices of the colo-vesical fistula (arrowed). 


\section{Discussion:}

Treatment of colonic diverticular disease has evolved over the past years. Most episodes are simple and can be successfully treated with antibiotics alone. For complicated diverticulitis, a strong trend is developing towards less invasive therapies including interventional radiology and laparoscopic lavage in an effort to avoid the morbidity and discomfort of a diverting colostomy. Based on a better understanding of the natural history of the disease, the indication for prophylactic colectomy after a few episodes of simple diverticulitis has been seriously challenged and for those patients who need a colectomy, laparoscopy is being proposed. ${ }^{10,11}$

Laparoscopic exploration detected variant indications for conversion to open surgery in four cases including multiple entero-colic fistulas that required multiple resections, involvement of other viscera in the phlegmon with abscess formation and multiple adhesions which hindered dissection; exploratory laparoscopy of these cases helped to change surgical decision to open laparotomy for completion with a conversion rate of $33.3 \%$. This high conversion rate could be attributed to factors associated with chronicity and complications which reflect the possibility of multiple recurrent acute attacks that resolved medically. In hand with such explanation Nguyen et al. ${ }^{12}$ reported a $36 \%$ conversion rate to open and that all conversions were due to dense pelvic adhesions and severe inflammation resulting in difficult dissections. Also, Coli et al. ${ }^{13}$ reported that patients with a history of abscess had a $23 \%$ chance of conversion, while those with no abscess history had an $8 \%$ chance of conversion. Natarajan et al. ${ }^{14}$ and Chouillard et al. ${ }^{15}$ attributed the higher rates of conversion to open surgery to operative circumstances without direct relationship to timing of surgery after an acute attack. Zinzindohou $\&$ Samama $^{16}$ documented that as the lesions of resected colon became more severe due to restricted indications, laparoscopic approach will require more surgical skill and conversion rate might increase. In selected cases, open surgery should be preferred
Three women had diverticulitis associated with gynecological pathology; two had left colosalpingeal fistula with amalgamation of the left ovary and underwent laparoscopic left salpingo-oophrectomy in addition to left colectomy, while the third patient had multiple colo-uterine and colo-vesical fistulas with left adnexal abscess including the left fallopian tube and left ovary and subtotal supra-vaginal hysterectomy including the left adnexal mass with the ovary and the tube was performed in addition to left colectomy. Preoperative diagnosis of colo-salpingeal fistulas relied on the CT findings that showed air and fluid collections in the adnexia, which coincided with Panghaal et al. ${ }^{17}$ who reported that in patients with diverticulitis, a collection of gas, either alone or in combination with fluid, within the adnexum is a sensitive and specific predictor of the presence of a fistula between the colon and an adnexum. Similar to the applied policy, Breitenstein et al. ${ }^{18}$ reported two women suffering from diverticulitis and symptomatic uterus myomatomas treated by combined laparoscopic sigmoid resection and laparoscopically assisted transvaginal hysterectomy. Also, Fernandez ${ }^{19}$ reported cases of tuboovarian abscess as a presentation for women with diverticulitis.

For both patients had colo-vesical fistula, the fistulous openings were identified and bladder wall was closed with inverting pursestring suture placed in healthy bladder tissue. Such simple closure coincided with Melchior et al. ${ }^{20}$ who managed patients with colovesical fistulas with resection of the fistulized bowel, single stage bowel anastomosis without protective colostomy and closure of the bladder defect. Urethral catheter removal is a point of controversy concerning early or late removal, the current study relied on performing cystogram before catheter removal and after assurance of absence of leakage; the catheter was removed. Such policy goes in hand and supported the findings of de Moya et al. ${ }^{21}$ who reported that patients with early Foley catheter removal did not have significant complications compared with patients with late Foley catheter removal after simple bladder repair of colo-vesical 
fistula secondary to diverticulitis.

Diverticular absceses were detected in 7 cases, all were drained with peritoneal lavage followed by primary resection and anastomosis; similarly, Di Stefano et al. ${ }^{22}$ managed 20 of 35 cases of diverticular abscess with primary colonic resection.

The higher frequency of abscess formation among studied cases indicated the misleading outcome of conservative management of uncomplicated cases of diverticulitis and point to the necessity of early surgical interference for uncomplicated cases to guard against the development of complications and dealing with the case as complicated diverticulitis. Such opinion coincided with Ricciardi et al. ${ }^{23}$ who retrospectively reported the following data: the ratio of diverticulitis discharges increased from 5.1 cases per 1,000 inpatients in 1991 to 7.6 cases per 1,000 inpatients in 2005 , but the proportion of patients who underwent colectomy for uncomplicated diverticulitis declined from $17.9 \%$ in 1991 to $13.7 \%$ in 2005 and during the same period, the proportion of free diverticular perforations as a fraction of all diverticulitis cases remained unchanged, but even the proportion of diverticular abscess as a fraction of all diverticulitis cases increased from 5.9\% in 1991 to $9.6 \%$ in 2005. In support of such opinion, Zdichavsky et al. ${ }^{24}$ reported that patients with acute diverticulitis receiving a laparoscopic early single-stage procedure benefited from an early postoperative convalescence with a minimum of disability.

The recorded operative and immediate postoperative data were acceptable and go in hand with $\mathrm{Li}$ et al. ${ }^{25}$ who compared laparoscopic-assisted versus open approach for management of complicated diverticulitis and found that laparoscopic-assisted group had significantly less blood loss with significantly shorter time to first bowel motion ( 3.5 vs. 5 days) in the laparoscopicassisted group.

In conclusion, laparoscopic management of complicated diverticulitis is feasible and safe approach provided that proper surgical decision was adequately taken concerning to proceed or not after laparoscopic exploration.
Laparoscopic approach for management of complicated diverticulitis allowed patients to enjoy all advantages of laparoscopic surgery as a minimally invasive surgery.

\section{References:}

1- Roberts P, Abel M, Rosen L: Practice parameters for sigmoid diverticulitis. The Standards Task Force American Society of Colon and Rectal Surgeons. Dis Colon Rectum 1995; 38: 125-132.

2- Chautems R, Ambrosetti P, Ludwig A: Long-term follow-up after first acute episode of sigmoid diverticulitis: Is surgery mandatory? Dis Colon Rectum 2001; 44: A5-A26.

3- Häring RU, Salm R: Sigmoid diverticulitis - indications for surgery and choice of procedure. MMW Fortschr Med 2003; 145(40): 32-35.

4- Patel NA, Bergamaschi R: Laparoscopy for diverticulitis. Semin Laparosc Surg 2003; 10(4):177-183.

5- Aydin HN, Remzi FH: Diverticulitis: When and how to operate? Dig Liver Dis 2004; 36(7): 435-445.

6- Ignjatovic D, Zivanovic V, Vasic G, Ilic I: Meta-analysis on minimally invasive surgical therapy of sigmoid diverticulitis. Acta Chir Iugosl 2004; 51(3): 25-28.

7- Whetsone D, Hazey J, Pofahl WE 2 ${ }^{\text {nd }}$, Roth JS: Current management of diverticulitis. Curr Surg 2004; 61(4): 361-365.

8- Chapman JR, Wolff BG: The management of complicated diverticulitis. Adv Surg 2006; 40: 285-297.

9- Teeuwen PH, chouten MG, Bremers AJ, Bleichrodt RP: Laparoscopic sigmoid resection for diverticulitis decreases major morbidity rates: A randomized controlled trial. Ann Surg 2009; 250(3): 500-501.

10-Di Mare L, Christoforidis D, Demartines $\mathrm{N}$ : Current treatment strategies for sigmoid diverticulitis. Rev Med Suisse 2010; 6(254): 1277-12781.

11-Hogan A, Winter D: Management of acute diverticulitis: Is less more? Dis Colon Rectum 2011; 54(1): 126-128.

12-Nguyen SQ, Divino CM, Vine A, Reiner M, Katz LB, Salky B: Laparoscopic surgery 
for diverticular disease complicated by fistulae. JSLS 2006; 10(2): 166-168.

13-Cole K, Fassler S, Suryadevara S, Zebley DM: Increasing the number of attacks increases the conversion rate in laparoscopic diverticulitis surgery. Surg Endosc 2009; 23(5): 1088-10892.

14-Natarajan S, Ewings EL, Vega RJ: Laparoscopic sigmoid colectomy after acute diverticulitis: When to operate? Surgery 2004; 136(4): 725-730.

15-Chouillard E, Benhaim L, Ata T, Etienne JC, Ghiles E, Fingerhut A: Elective laparoscopic colectomy in uncomplicated diverticulitis: When should surgery be performed? Cir Esp 2007; 81(4): 207-212.

16-Zinzindohou F, Samama G: Colonic diverticulosis: Which patients need surgery? Rev Prat 2009; 59(1): 16-19.

17-Panghaal VS, Chernyak V, Patlas M, Rozenblit AM: CT features of adnexal involvement in patients with diverticulitis. AJR Am J Roentgenol 2009; 192(4): 963-966.

18-Breitenstein S, Dedes KJ, Bramkamp M, Hess T, Decurtins M, Clavien PA: Synchronous laparoscopic sigmoid resection and hysterectomy with transvaginal specimen removal. $J$ Laparoendosc Adv Surg Tech A 2006; 16(3): 286-289.

19-Fernández García N, Mesa Álvarez A, Calvo Blanco J, Alvarez Vázquez A, Pereira Menedez C, Benítez Vázquez AM: Tuboovarian abscess as unusual presentation of tubarian fistula secondary to sigmoid diverticulitis. Rev Esp Enferm Dig 2011; 103(5): 264-265.

20-Melchior S, Cudovic D, Jones J, Thomas C, Gillitzer R, Theoff J: Diagnosis and surgical management of colovesical fistulas due to sigmoid diverticulitis. J Urol 2009; 182(3): 978-982.

21-de Moya MA, Zacharias N, Osbourne A, Butt MU, Alam HB, King DR, McGovern F, Velmahos GC: Colovesical fistula repair: Is early Foley catheter removal safe? $J$ Surg Res 2009; 156(2): 274-277.

22-Di Stefano G, Zanghì GN, Biondi A, Benfatto G, Basile F: Surgical treatment of acute complicated diverticulitis. G Chir 2009; 30(8-9): 355-358.

23-Ricciardi R, Baxter NN, Read TE, Marcello PW, Hall J, Roberts PL: Is the decline in the surgical treatment for diverticulitis associated with an increase in complicated diverticulitis? Dis Colon Rectum 2009; 52(9): 1558-1563.

24-Zdichavsky M, Königsrainer A, Granderath FA: Laparoscopic rectosigmoid resection for acute sigmoid diverticulitis. $J$ Gastrointest Surg 2009; 13(4): 804-805.

25-Li JC, Ng SS, Lee JF, Yiu RY, Hon SS, Leung WW, Leung KL: Emergency laparoscopic-assisted versus open right hemicolectomy for complicated cecal diverticulitis: A comparative study. $J$ Laparoendosc Adv Surg Tech A 2009; 19(4): 479-483. 